\title{
Supply Chain Coordination Strategy Considering Manufacturer Quality Improvement
}

\author{
Hongqi Wang ${ }^{1, *}$ \\ ${ }^{1}$ School of Business, Xinhua College of Sun Yat-Sen University, Guangzhou Guangdong 510520, China \\ *Corresponding author. Email: wanghongqi1001@163.com
}

\begin{abstract}
Based on manufacturer quality improvement, this paper analyzes the optimal strategies under centralized and decentralized decision-making, and further studies the relationship between quality improvement degree and retail price, sales and profit. the results show that the centralized decision-making and decentralized decision making, quality improvement degree of price and retail price change in the opposite direction. The influence factor of quality improvement demand is small, and the degree of quality improvement changes positively with order quantity and profit. When the quality improvement demand impact factor is greater than the cost impact factor, it is beneficial to adjust the order quantity and supply chain profit to achieve the optimal.
\end{abstract}

Keywords: product quality improvement, coordination strategy, game theory

\section{INTRODUCTION}

With the rapid development of e-commerce, the quality of online goods is gradually inferior. Therefore, the quality of online goods is becoming more and more prominent. Quality supervision departments, major e-commerce platforms and other related industries have taken various measures to strengthen the management of quality problems, but the effect is very small. Many scholars began to improve the quality of network goods by micro research.

Some scholars focus on the relationship between service level and product quality. For example, Banker [1] analyzed the equilibrium level of product quality under different competition levels. Lu, Shi etal. studied how product quality affects the balance and performance of the supply chain during the operation of the supply chain [2,3], and Xiao etal. [4] studied the coordination strategy of the supply chain under the dual competition of quality and price in a secondary supply chain composed of a manufacturer and two suppliers. Li etal. [5] optimized product quality and retail pricing when considering the fairness concerns of members of the supply chain. Lin etal. [6] studied manufacturers' product quality level selection and pricing strategies under different channels, as well as the influence of equilibrium results on product quality level, product price, manufacturers' and retailers' profits. In a word, the above measures to improve product quality mainly focus on achieving the balance of product quality and price through the coordination of supply chain. The quality improvement measures taken by manufacturers under market pressure and policy factors are not discussed separately. Therefore, this paper considers the manufacturers' active quality improvement strategies and further analyzes the impact of quality improvement on retail prices, sales and profits organization of the text.

\section{BASIC MODEL AND HYPOTHESIS}

This study is based on a two-tier supply chain led by a single manufacturer, followed by a single retailer, and the degree of quality improvement is the research parameter for the coordination of supply chain. The meanings of each parameter are shown in table 1 . We made the following assumptions:

Table 1 Notations

\begin{tabular}{|c|c|}
\hline Parameter & Definition \\
\hline$a$ & market capacity \\
\hline$\theta$ & coefficient of price elasticity \\
\hline$\varphi$ & quality complaint rate improvement \\
\hline$w$ & wholesale price \\
\hline$c$ & manufacturer's initial cost \\
\hline$p$ & sales price \\
\hline$m$ & cost sensitivity to quality improvement \\
\hline$k$ & consumer sensitivity to quality \\
\hline
\end{tabular}

(1) Assume that there are no unmarketable goods. $c$ is the manufacturer's initial production cost? $\varphi^{-m}$ refers to the production cost of the manufacturer after quality improvement, where $m>0$, the higher the value is, the greater the impact on the cost is.

(2) Consumers have certain requirements for product quality and are willing to pay higher prices for highquality products. $\varphi^{k}$ represents the impact of quality improvement on demand, where $(k>0)$ it represents the impact factor of quality improvement demand.

Based on the above assumptions and parameters, the demand function can be obtained:

$$
Q=a-b p-\varphi^{k} p
$$


In order to distinguish the profit function of manufacturer, retailer and the whole supply chain, the variables $m, r, s c$ are used as the subscripts respectively. Then the respective profit function can be obtained:

$$
\begin{aligned}
& \pi_{r}=\left(a-b p-\varphi^{k} p\right)(p-w) \\
& \pi_{m}=\left(a-b p-\varphi^{k} p\right)\left(w-c \varphi^{-m}\right) \\
& \pi_{s c}=\left(a-b p-\varphi^{k} p\right)\left(p-c \varphi^{-m}\right)
\end{aligned}
$$

\section{MODEL ANALSIS}

\subsection{Manufacturer does not improve quality level}

\section{1 .1 centralized decision-making}

Manufacturers and retailers constitute a virtual entity, which is regarded as a decision-making body. The profits of the whole supply chain under the centralized decision are as follows: $\pi_{s c}=(a-b p)(p-c)$, And we can solve it:

$$
\begin{aligned}
p & =\frac{a+b c}{2 b} \\
Q & =\frac{a-b c}{2} \\
\pi_{s c} & =\frac{(a-b c)^{2}}{4 b}
\end{aligned}
$$

The price-cost ratio is:

$$
\beta_{1}=\frac{a}{2 b c}+\frac{1}{2}
$$

\subsection{2 decentralized decision-making}

Combined with equations (2) and (3), it can be obtained that:

$$
\begin{aligned}
& p=\frac{3 a+b c}{4 b} \\
& Q=\frac{a-b c}{4} \\
& w=\frac{a+b c}{2 b}
\end{aligned}
$$

Put $p, Q, w$ into formula (2), (3) and (4) to obtain the profits of the manufacturer, the retailer and the whole supply chain:

$$
\pi_{r}=\frac{(a-b c)^{2}}{16 b}
$$

$$
\begin{aligned}
& \pi_{m}=\frac{(a-b c)^{2}}{8 b} \\
& \pi_{s c}=\frac{3(a-b c)^{2}}{16 b}
\end{aligned}
$$

The price-cost ratio is:

$$
\beta_{2}=\frac{a}{4 b c}+\frac{1}{4}
$$

Since $Q$ is greater than zero, it can be known that: $1<\beta_{1}<\beta_{2}$, according to the above expression, the price-cost ratio is affected by the market size, the manufacturer's initial cost and the price sensitivity coefficient.

\section{2 manufacturers improve the quality level}

\subsection{1 centralized decision-making}

According to formula (4), find the first and second derivatives of $\pi_{s c}$ with respect to $p$ :

$$
\begin{gathered}
\frac{\partial \pi_{s c}}{\partial p}=-2\left(b+\varphi^{k}\right) p+a+\left(b+\varphi^{k}\right) c \varphi^{-m} \\
\frac{\partial^{2} \pi_{s c}}{\partial p^{2}}=-2\left(b+\varphi^{k}\right)<0
\end{gathered}
$$

As the second derivative above is less than zero, it can be known that there is a maximum value of $\pi_{s c}$. Then, if the first derivative is zero, it can be obtained as follows:

$$
\begin{gathered}
p=\frac{a+\left(b+\varphi^{k}\right) c \varphi^{-m}}{2\left(b+\varphi^{k}\right)} \\
Q=\frac{a-\left(b+\varphi^{k}\right) c \varphi^{-m}}{2} \\
\pi_{s c}=\frac{\left[a-\left(b+\varphi^{k}\right) c \varphi^{-m}\right]^{2}}{4\left(b+\varphi^{k}\right)}
\end{gathered}
$$

Proposition 1: under centralized decision-making, retail price is a monotonic function of the level of quality improvement.

Proving Course: The first derivative of $p$ with respect to $\varphi$ is:

$$
\frac{\partial p}{\partial \varphi}=-\frac{a k \varphi^{k}}{2\left(b+\varphi^{k}\right)^{2}}-\frac{c m}{2 \varphi^{m+1}}<0
$$

So the retail price is a monotonic function of the level of quality improvement.

\section{Proposition 2:}

1) when $m / k<1$, there is an optimal solution for the optimal order quantity 
2) When $1<m / k<\beta_{1}$, the optimal order quantity is a monotone increment function of the quality improvement degree.

3) when $m / k>\beta_{1}$, the manufacturer does not take quality improvement measures, and the order quantity is the same.

\section{Proving Course:}

1) The first and second derivatives of $Q$ with respect to $\varphi$ can be obtained as follows:

$$
\begin{gathered}
\frac{\partial Q}{\partial \varphi}=\frac{c}{2 \varphi^{m+1}}\left[m b+(m-k) \varphi^{k}\right] \\
\frac{\partial^{2} Q}{\partial \varphi^{2}}=-\frac{c}{2 \varphi^{m+2}}\left[(k-m) k \varphi^{k}+m(m+1)\left(b+\varphi^{k}\right)\right]<0
\end{gathered}
$$

when $m / k<1$, So if the second derivative is less than zero, there is an optimal solution for the order quantity. Further solutions can be obtained as follows:

$$
\varphi^{*}=\sqrt[k]{m b /(k-m)}
$$

2) When $1<m / k<\beta_{1}$, it can be known from the process of proof 1 that $\partial Q / \partial \varphi>0$, Then the order quantity is the monotone increment function of the quality improvement degree.

3) $\beta_{1}$ is the price-cost ratio of manufacturing without taking quality improvement measures. $m / k$. refers to the ratio of the extra cost paid to the trigger demand factor when improving quality, when $m / k>\beta_{1}$, It means that the cost ratio paid to improve the quality of goods is greater than the benefit without improvement, which will eventually reduce the total utility. As a rational decision maker, manufacturers will choose the strategy without improvement.

\subsection{2 decentralized decision-making}

According to equation (2), the first and second derivatives of $\pi_{r}$ with respect to $p$ can be obtained:

$$
\begin{gathered}
p=\frac{3 a+\left(b+\varphi^{k}\right) c \varphi^{-m}}{4\left(b+\varphi^{k}\right)} \\
w=\frac{a+\left(b+\varphi^{k}\right) c \varphi^{-m}}{2\left(b+\varphi^{k}\right)} \\
Q=\frac{a-\left(b+\varphi^{k}\right) c \varphi^{-m}}{4}
\end{gathered}
$$

Put $p, Q, w$ into formula (2), (3) and (4) to obtain the profits of the manufacturer, the retailer and the whole supply chain:

$$
\begin{aligned}
& \pi_{r}=\frac{\left[a-\left(b+\varphi^{k}\right) c \varphi^{-m}\right]^{2}}{16\left(b+\varphi^{k}\right)} \\
& \pi_{m}=\frac{\left[a-\left(b+\varphi^{k}\right) c \varphi^{-m}\right]^{2}}{8\left(b+\varphi^{k}\right)} \\
& \pi_{s c}=\frac{3\left[a-\left(b+\varphi^{k}\right) c \varphi^{-m}\right]^{2}}{16\left(b+\varphi^{k}\right)}
\end{aligned}
$$

Proposition 3: both wholesale and retail prices are monotonic subtractions of the level of quality improvement under decentralized decision making.

\section{Proving Course:}

The first derivative of $p, w$ with respect to $\varphi$ can be obtained:

$$
\begin{aligned}
& \frac{\partial w}{\partial \varphi}=-\frac{a k \varphi^{k}}{2\left(b+\varphi^{k}\right)^{2}}-\frac{c m}{2 \varphi^{m+1}}<0 \\
& \frac{\partial p}{\partial \varphi}=-\frac{3 a k \varphi^{k}}{3\left(b+\varphi^{k}\right)^{2}}-\frac{c m}{4 \varphi^{m+1}}<0
\end{aligned}
$$

Since the first derivatives above are all less than zero, the wholesale price and retail price can be obtained as a minus function of the degree of quality improvement.

Proposition 4: when making decentralized decisions, manufacturers' profits will be different under different cost-demand ratios, which will affect the quality improvement strategy.

1) When $m / k>\beta_{2}$, the manufacturer abandoned the improvement strategy due to the high cost of quality improvement.

2) When $1<m / k<\beta_{2}$, the manufacturer continues to improve the quality, and there is an optimal level of improvement.

\section{Proving Course:}

1)When $m / k>\beta_{2}$, according to the proof process of proposition 2 , the manufacturer will give up the quality improvement strategy because the cost of quality improvement is much higher than the resulting benefit.

2) Find the first and second derivatives of $\pi_{m}$ with respect to $\varphi$ :

$$
\begin{aligned}
& \frac{\partial \pi_{m}}{\partial \varphi}=\frac{k \varphi^{k-1}}{8\left(b+\varphi^{k}\right)^{2}}\left[c^{2} \varphi^{-2 m}\left(b+\varphi^{k}\right)^{2}-a^{2}\right] \\
& -\frac{c m \varphi^{-m-1}}{4}\left[\left(c \varphi^{-m}\left(b+\varphi^{k}\right)-a\right)\right]
\end{aligned}
$$




$$
\begin{aligned}
& \frac{\partial^{2} \pi_{m}}{\partial \varphi^{2}}=-\frac{c^{2} \varphi^{k-2 m-2}\left[4 m^{2}+2 m+k(k+1)-4 m k\right]}{8} \\
& \frac{-a^{2} k \varphi^{k-2}(k+1)}{8\left(b+\varphi^{k}\right)^{2}}+\frac{c m \varphi^{-2 m-2}(c b-a)(m+1)}{4}
\end{aligned}
$$

When $m>k, 4 m^{2}+2 m+k(k+1)-4 m k>0$, Because in the absence of quality improvement by the manufacturer, to ensure that the order quantity is greater than zero, thus known $b c-a<0$, so $\partial^{2} \pi_{m} / \partial \varphi^{2}<0$, There is a maximum value for $\pi_{m}$.

\section{NUMERICAL SIMULATION}

To further elaborate the proof process, this section will analyze based on the assignment of parameters. It is assumed that the value of the parameter is as follows: $a=120, b=5, c=2, m=1, k=0.5$.

(1) Study on the relationship between retail price and quality improvement degree.

Substitute the parameter value, according to the above relation, the subtraction function of the improvement degree of retail price quality can be found through numerical simulation, as shown in figure 1. Similarly, it can be proved that the price under decentralized decision is also a function of the degree of quality improvement. This is the same conclusion as propositions 1 and 3 .

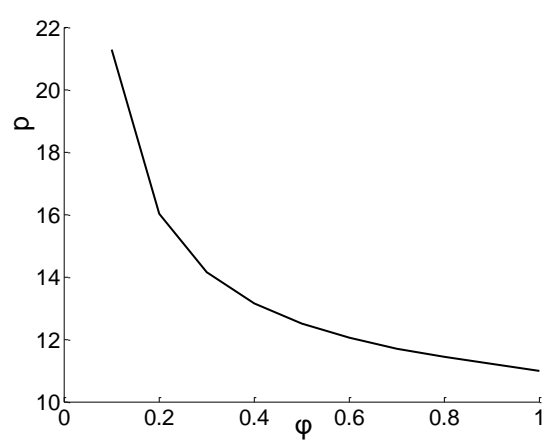

Figure 1 The effect of $\varphi$ on $p$

(2) Study on the relationship between order quantity and quality improvement degree.

1) When $m>k$, the order quantity will continue to increase. The order quantity is an increment function of the degree of quality improvement. The simulation results are shown in figure 2 by substituting the parameters into the formula.

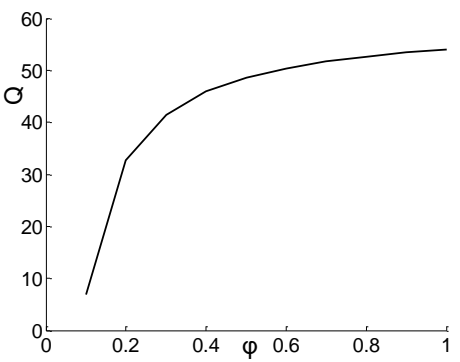

Figure 2 The effect of $\varphi$ on $Q$

2) When $m<k$, let $\varphi=0.8, k \in(10,20), m \in(0,10)$, and substitute in other known parameters to simulate different combinations, as shown in figure 3 . It can be seen from figure 3 that the order quantity increases with the increase of the demand impact factor and decreases with the increase of the quality improvement cost factor. In different combinations, there is an optimal order quantity. This is the same conclusion as propositions 2 .

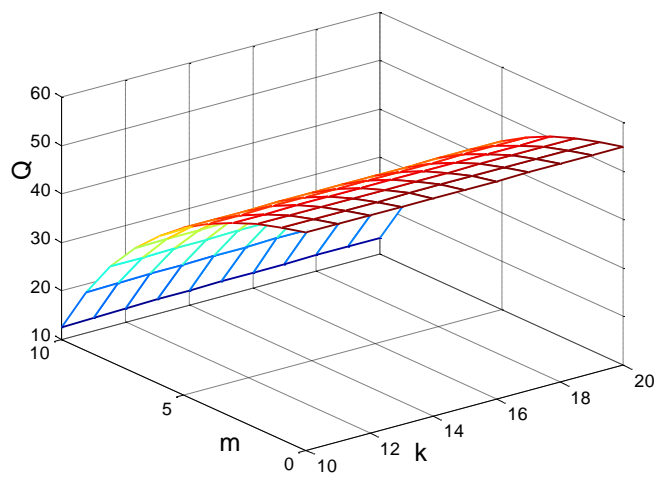

Figure 3 The effect of $m, k$ on $Q$

(3) The relationship between profit and the degree of quality improvement.

Since the overall profit of manufacturer, retailer and supply chain varies in the same way, the manufacturer's profit is selected as an example.

1) when $m>k$, the manufacturer's profit is substituted into the known parameters, and the simulation results are shown in figure 4 . Therefore, it can be known that $\pi_{m}$ is a monotonically increasing function of $\varphi$, which is the same as the conclusion of proposition 4 .

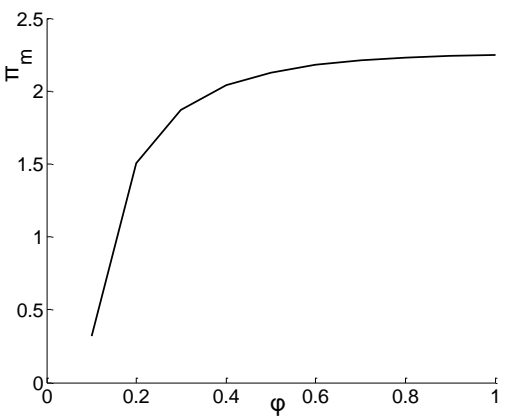

Figure 4 The effect of $\varphi$ on $\pi_{m}$ 
2) when $m<k$, also assume that $\varphi=0.8, k \in(10,20)$, $m \in(0,10)$ Substitute in other known parameters to show the effect on the manufacturer's profits as shown in figure 5.

As can be seen from figure 5, there is a positive relationship between supply chain and member profits and demand impact factors. And the quality improvement cost factor into the reverse change relationship. In the different combinations of $m, k$, there is the combination point with the greatest profit.

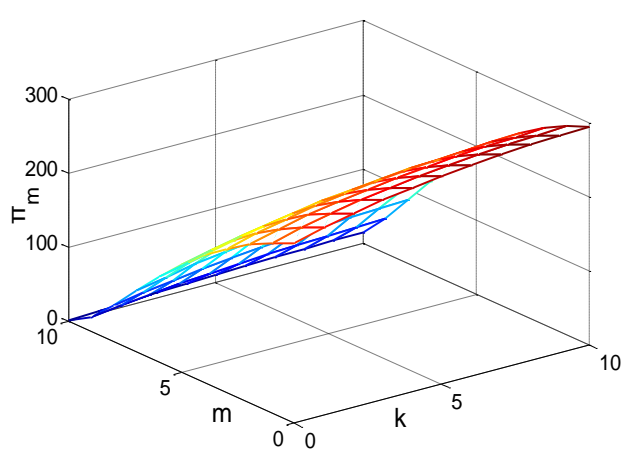

Figure 5 The effect of $m, k$ on $\pi_{m}$

\section{SUMMARY}

The effect of quality improvement on sales price, order quantity and profit is studied on the basis of quality improvement of manufacturer. The results show that whether the decision is centralized or decentralized, the degree of quality improvement has a negative effect on the selling price and the wholesale price. Further research shows that when the demand impact factor for quality improvement is less than the cost impact factor, the degree of quality improvement has a positive effect on order quantity and profit. When the quality improvement demand impact factor is greater than the cost impact factor, the profit and order quantity have the optimal value.

This paper only considers the manufacturer's initiative to improve product quality, and does not consider the cost allocation in the process of quality improvement. Meanwhile, it can also further study the cost allocation under random demand.

\section{ACKNOWLEDGMENT}

This work was supported by Scientific Research Project Funds of Guangdong University Provincial Youth (GrantNO.2018WQNCX277), Scientific research project Funds of Xinhua College of sun yat-sen university (Grant NO. 2018YB011).

\section{REFERENCES}

[1] Banker, K. Quality and competition, Management Science, 44(9) (1998) 1179-1192. DOI: https://doi.org/10.1287/mnsc.44.9.1179.

[2] Qihui Lu, Daoli Zhu. Research on equilibriums and coordination strategies of supply chains with quality and price competition, Journal of Management Sciences in China, 12(3) (2009) 56-66. DOI: https://doi.org/10.3321/j.issn:1007-9807.2009.03.006

[3] Kui-ran Shi, Hujie Ma, Yuling Sun. Study on Evolution of the Manufacturers' Quality Investment and the Retailers' Trust, Soft Science, 12(28) (2014) 95-98. DOI: https://doi.org/10.3969/j.issn.10018409.2014.12.021.

[4] Di Xiao, Jingxia Yuan, Xing Bao. Supply Chain Coordination Strategy Considering Dual Competition from Price and Quality, Chinese Journal of Management Science, 21(4) (2013) 82-88. DOI: https://doi.org/10.16381/j.cnki.issn1003207x.2013.04.022

[5] Jicai Li, Yongwu Zhou, Changwen Li. Quality and retail pricing decision in a supply chain with fairness concerns, China Soft Science, 31(3) (2017) 139-144. DOI: https://doi.org/10.13956 /j.ss.1001 8409.2017.03.30

[6] Jing Lin, Jian Wang. Research of Manufacturers 'Channel Strategy under Dual-channel Supply Chain Based on Differentiated Product, Chinese Journal of Management Science, 26(6) (2018) 72-84. DOI: https://doi.org/10.16381/j.cnki.issn1003207x.2018.06.008. 